\title{
Evidence for divided automatic attention
}

\author{
P.S. Silva and L.E. Ribeiro-do-Valle \\ Departamento de Fisiologia e Biofísica, Instituto de Ciências Biomédicas, Universidade de São Paulo, \\ São Paulo, SP, Brasil \\ Correspondence to: L.E. Ribeiro-do-Valle, Departamento de Fisiologia e Biofísica, ICB, USP, 05508-000 \\ São Paulo, SP, Brasil \\ E-mail: ribeiro@icb.usp.br
}

\begin{abstract}
A long-standing debate in the literature is whether attention can form two or more independent spatial foci in addition to the wellknown unique spatial focus. There is evidence that voluntary visual attention divides in space. The possibility that this also occurs for automatic visual attention was investigated here. Thirty-six female volunteers were tested. In each trial, a prime stimulus was presented in the left or right visual hemifield. This stimulus was characterized by the blinking of a superior, middle or inferior ring, the blinking of all these rings, or the blinking of the superior and inferior rings. A target stimulus to which the volunteer should respond with the same side hand or a target stimulus to which she should not respond was presented 100 ms later in a primed location, a location between two primed locations or a location in the contralateral hemifield. Reaction time to the positive target stimulus in a primed location was consistently shorter than reaction time in the horizontally corresponding contralateral location. This attentional effect was significantly smaller or absent when the positive target stimulus appeared in the middle location after the double prime stimulus. These results suggest that automatic visual attention can focus on two separate locations simultaneously, to some extent sparing the region in between.
\end{abstract}

Key words: Automatic attention; Divided attention; Visual priming; Go/no-go reaction time

Research supported by CNPq and FINEP. Publication supported by FAPESP.

Received May 28, 2007. Accepted January 3, 2008

\section{Introduction}

People usually explore their environment by focusing their attention on only one stimulus at a time. The strategy of spreading one's attention to encompass several stimuli is more rarely used, because it results in poor perception of these stimuli, presumably due to the mutual interference of their neural representations (see Ref. 1).

There are circumstances, however, in which it is important to follow two (or more) stimuli at the same time; for example, when playing soccer, a game in which a player has to follow the movements of two or more opposition and/or home team players. If these stimuli are very close together in space, attention could spread to cover the area they occupy. However, if they are separated by some distance, this strategy would not be the best option, since intervening stimuli would also be processed together with the relevant stimuli and compete with them. This gives rise to the question whether attention would be divided be- tween the separate locations in such a case. The intuitive notion is that this would not be possible. It is difficult to imagine ourselves trying to scan two or more locations using such a strategy.

Supporting this impression, several studies have presented evidence that voluntary attention cannot be split. One of the initial studies was performed by Posner et al. (2) who asked their volunteers to simultaneously attend to two of four locations aligned with the horizontal meridian, two in the left visual hemifield and two in the right visual hemifield, and to respond to the blinking of a light-emitting diode in one of these locations. One of the locations that should be attended was indicated by a central symbolic cue on a trialby-trial basis; the target stimulus appeared there in $65 \%$ of the trials. The other location that should be attended was fixed along each block of trials; the target stimulus appeared in this location in $25 \%$ of the trials. The remaining two locations could contain the target stimulus with a probability of $5 \%$ each. When the two most frequent loca- 
tions were adjacent, reaction time for the second most likely location was similar to that for the most likely location and shorter than those for the two less likely locations. When the two most frequent locations were separated by a less frequent location, reaction time for the second most likely location was longer than for the most likely one and similar to those for the less likely locations. These findings were taken as evidence that as far as target stimulus detection is concerned, attention splitting is not possible. McCormick and Klein (3) instructed their volunteers by means of a symbolic central cue to pay attention to one location or two non-adjacent same hemifield locations, of six equidistant locations aligned with the horizontal meridian, three on each side of fixation. In $80 \%$ of the trials a target stimulus appeared some time later, at an indicated location (56\% of the trials) or at a non-indicated location ( $24 \%$ of the trials). Reaction time to the target stimulus at the middle location on one side did not differ between the condition in which this same location was cued and the condition in which the external and the inner locations on the same side were cued, suggesting that the participants adopted the strategy of attending to the mid-location when cued to two non-adjacent locations. In another study, McCormick et al. (4) obtained additional evidence supporting this idea. In a first experiment there were two marked locations, one on each side of fixation. One or both locations were cued. Five hundred and fifteen milliseconds later, a target stimulus was presented at one of these locations or at one of two probe locations between the marked locations and fixation. Reaction time to the target stimulus in the probe location did not differ between the one-cued location condition and the two-cued location condition. In a second experiment, there were four marked locations, two in each hemifield, one near and one far from fixation. One of the far locations, the two far locations or all four locations were cued. The target stimulus was presented at one of the four locations. Reaction time to the target stimulus in a near fixation location did not differ between the two-cued location condition and the four-cued location condition. Recording event-related brain potentials, Heinze et al. (5) verified that probe stimuli presented at a non-cued location between two cued locations evoked responses similar to those evoked by probe stimuli presented at cued locations and greater than those evoked by probe stimuli presented at a non-cued location outside the zone delimited by the two-cued locations. The fact that irrelevant information arising from the intervening noncued location was treated as that coming from a cued location provides additional support for the idea that when attention must be directed to two non-adjacent locations it spreads to also encompass the area between them.
Some investigators, however, claimed to have observed a spatial division of attention. Müller and Findlay (6) tested the capacity of their volunteers to simultaneously attend to two of four equally excentric locations. One of the relevant locations was cued by a long central line as more likely to contain the impending target stimulus and the other relevant location was cued by a short central line as less likely to contain the impending target stimulus. The two non-cued locations were least likely to contain the impending target stimulus. The authors found that sensitivity was lower for the location cued as less likely than for the location cued as more likely, and was even lower for the uncued locations. These findings were interpreted as indicating that attention can be independently controlled at two separate locations. A subsequent study by Castiello and Umiltà (7) obtained results that could be considered to point in the same direction. A target stimulus was presented inside one of two square boxes of different sizes located to the left and to the right of fixation. In $45 \%$ of the trials, a central arrow correctly indicated the box where the target stimulus would appear. In $22.5 \%$ of the trials, the central arrow incorrectly indicated the other box, and in $22.5 \%$ of the trials, a nondirectional cross indicated that the target stimulus could appear in either box. As expected, reaction times were shorter in the valid condition than in the neutral condition and longer in the invalid condition than in the neutral condition. More importantly, both in the valid condition and in the neutral condition reaction times increased with the size of the box where the target stimulus appeared, suggesting that the efficiency of attention decreases with covered area and can be different at two separate locations. In fact, the results of Müller and Findlay (6) and Castiello and Umiltà (7) do not represent direct proof of a division of attention in space, although the apparently independent control of attention in two locations that they observed could be taken as an indication that such a division might be possible.

There are two studies whose results support the idea that attention can divide in space, at least under certain conditions. Egly and Homa (8) asked their subjects to identify a central letter and at the same time to locate another letter that could appear in 24 peripheral positions (eight equidistant positions $1^{\circ}$ far from fixation, eight equidistant positions $2^{\circ}$ far from fixation and eight equidistant positions $3^{\circ}$ far from fixation). In valid trials, the ring where the peripheral target letter would appear was correctly indicated. In invalid trials, another ring than that where the peripheral target letter would appear was incorrectly indicated. In neutral trials, no indication was given about the ring where the peripheral target letter would appear. Independent of the ring that was cued, the mean error percent- 
age in locating the peripheral target letter was lower in the valid condition as compared to the neutral condition, and lower in this condition than in the invalid condition. These results suggest that the observers were selectively attending to the regions located in the cued ring. In other words, attention would be able to be directed to spatially separate regions without including intervening regions. More recently, Awh and Pashler (9) conducted a series of experiments in which they specifically tested the hypothesis that attention could be spatially divided. Two horizontally or vertically oriented locations were cued. In $80 \%$ of the trials two target numbers were presented at the cued locations. In $20 \%$ of the trials one target number was presented directly in between the cued locations and another target number at a far location. Twenty-three distractor letters surrounded these target numbers. Accuracy was lower for the target number in between the cued locations than for the target numbers in these locations. Accuracy was even lower for the target number in the far location. When the distractor letters were eliminated, the accuracy at the middle location (and at the far location) increased substantially. The most parsimonious explanation for these findings is that attention was split between the two cued locations, inhibiting sensory processing at the intermediary and far regions.

Physiological evidence supporting the idea that attention can be divided in space was presented by Müller et al. (10) and McMains and Somers (11). Müller et al. (10) recorded frequency-coded steady-state visual evoked potentials to stimuli presented concurrently at four locations along the horizontal meridian. The observers were instructed to orient their attention to two adjacent locations, either in the left or right visual field, or two nonadjacent locations, a far location in one visual field and a near location in the other visual field. The amplitude of the evoked potential elicited by the stimulus presented at the location in between the two indicated nonadjacent locations was smaller than that of the evoked potentials elicited by the stimuli presented at these nonadjacent locations or that of the evoked potentials elicited by the stimuli presented at the indicated adjacent locations. It did not differ from the amplitude of the evoked potential elicited by the stimulus presented in the location in the hemifield opposite to the indicated adjacent locations. McMains and Somers (11) measured blood oxygenation level-dependent (BOLD) signal increase in visual areas (V1, V2, V3, V3A, and hV4) during the performance of a modified rapid, serial, visual presentation task. Volunteers were presented with five simultaneous streams of letters, one centrally (in the fixation point) and the others peripherally (one in each visual field quadrant). They were instructed to pay attention to the superior left stream, the superior left and central streams, the superior left, central and inferior right streams, or the superior left and inferior right streams. BOLD signal activation in the visual regions corresponding to the attended locations declined progressively from the first condition to the third. As expected, BOLD signal activation in the visual regions corresponding to the attended locations in the fourth critical condition was smaller than that corresponding to the attended location in the first condition. More important, it was not different from that obtained for the visual regions corresponding to the attended locations in the second condition and was greater than that obtained for the visual regions corresponding to the attended locations in the third condition. Equally important, BOLD signal activation in the visual region corresponding to the central location in the fourth critical condition was not different from that obtained for the visual region corresponding to a non-attended location. Accuracy in identifying the occurrence of the target letter in the attended streams of letters followed the same general pattern as the BOLD signal activation.

In the investigations just discussed, voluntary attention was tested. It is not known whether automatic attention, which is mobilized at short time intervals by peripheral prime stimuli, behaves in the same way as voluntary attention. The objective of the present study was to examine the possibility of a spatial division of this other kind of attention. The attentional effect obtained for the middle location of a double peripheral prime stimulus was compared with that produced by a single peripheral prime stimulus. A smaller effect elicited by the double prime stimulus, as compared to the single prime stimulus, would indicate that automatic attention can be divided in space, like voluntary attention.

The Ethics Committee of the Instituto de Ciências Biomédicas of Universidade de São Paulo approved this study and written informed consent was obtained from all participants.

\section{Experiment 1}

A robust attentional effect occurs when a single peripheral prime stimulus is presented on the horizontal meridian and a target stimulus is presented $100 \mathrm{~ms}$ later at the same location or at a symmetric location in the opposite hemifield (12). If the single prime stimulus is replaced by a double prime stimulus, whose components are located some degrees above and below the horizontal meridian, and the target stimulus is still presented on the horizontal meridian, two distinct alternative results could be obtained: either the attentional effect is similar to that occurring when a single prime is presented or it presents a marked decrease, 
perhaps even disappearing. The first result would indicate that automatic attention continues to form a unique focus, although with a greater spread. The second result would suggest that automatic attention can divide spatially, forming two independent focuses. To distinguish more clearly between the possibilities of a division of attention and a spread of attention, in case of a reduction in the attentional effect, a triple prime stimulus, with its components above, below and on the horizontal meridian, could also be used. With such a stimulus, automatic attention should form a unique spread focus or three independent foci. Finding a stronger attentional effect than that obtained with the double prime stimulus would support the division of attention hypothesis.
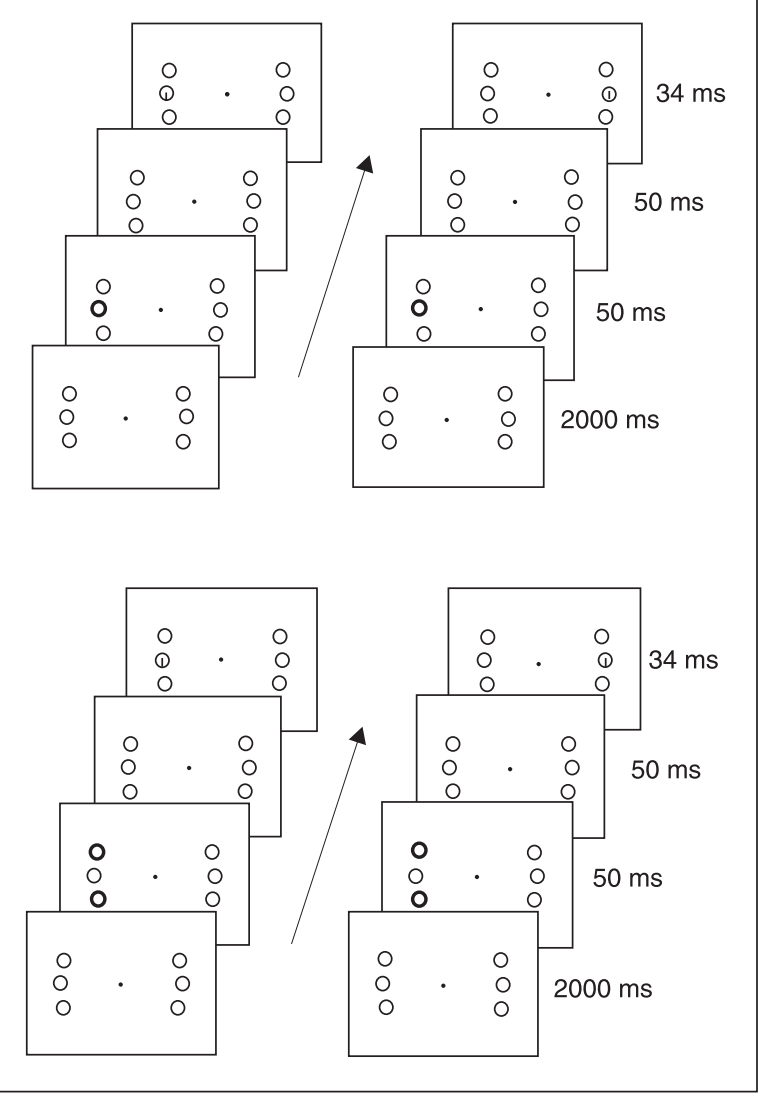

Figure 1. Illustration of the sequence of events in a single prime stimulus trial (upper panel) and a double prime stimulus trial (lower panel). The left side sequence of frames shows the positive target stimulus appearing on the primed hemifield and the right side sequence of frames shows it appearing in the opposite hemifield. Numbers on the right side of each panel refer to the duration (in milliseconds) of each frame. The message frame, that appeared immediately after the response and lasted 200 $\mathrm{ms}$, is not shown.
In the current experiment, the attentional effect produced by a double prime stimulus was compared to those produced by a single and a triple prime stimulus. A choice go/no-go reaction time task was employed to test the volunteers. The target stimuli were always presented in a middle location.

\section{Methods}

Participants. Twelve young adult females voluntarily participated in the experiment. All were right handed (as evaluated by the Edinburgh Inventory; 13), presented normal or corrected-to-normal vision and normal color vision. None of them had had previous experience with reaction time tasks or was aware of the purpose of the study.

Apparatus. The volunteers were tested in a dimly illuminated $\left(<0.1 \mathrm{~cd} / \mathrm{m}^{2}\right)$ and sound-attenuated room. Their head was spatially positioned by a chin-and-front rest so that their eyes stayed $57 \mathrm{~cm}$ away from the screen of a 15inch video monitor. The background color of this screen was white and its luminance was $27 \mathrm{~cd} / \mathrm{m}^{2}$. A central black square 0.05 degree wide and having a luminance of less than $0.01 \mathrm{~cd} / \mathrm{m}^{2}$ (fixation point, FP) and peripheral visual stimuli were presented on this screen. The volunteers were instructed to keep their eyes on the FP and to respond to some of these peripheral stimuli by pressing two keys, one on each side, with their index fingers. An IBM-compatible computer, controlled by programs developed with the MEL2 software (Psychology Software Tools Inc., Pittsburgh, USA), generated the stimuli and recorded the responses.

Procedure. Each volunteer participated in two testing sessions on separate days, not more than 7 days apart. Before each session she received a brief written explanation about the task to be performed. A more detailed explanation was given in the testing room while she was doing some example trials. The volunteer was then asked to do about 20 practice trials.

The main purpose of the first testing session was familiarizing the volunteers with the experimental conditions. It consisted of one block of 72 trials. Each trial began with the appearance of the FP and three light gray rings (1.5 degree in diameter and a 0.04-degree wide margin, with a luminance of $9 \mathrm{~cd} / \mathrm{m}^{2}$ ) on each side, at an excentricity of 9 degrees. The middle rings were located on the horizontal meridian. The center-to-center distance of the rings on each side was 3.5 degrees (see Figure 1). After 1850 to $2350 \mathrm{~ms}$, either the middle ring (single condition), the superior, middle and inferior rings (triple condition), or the superior and inferior rings (double condition) on one side got darker (luminance of $1 \mathrm{~cd} / \mathrm{m}^{2}$ ) for $50 \mathrm{~ms}$. Each one of these stimuli (the S1) occurred in one third of the trials, 
$50 \%$ of the trials on the left side and $50 \%$ of the trials on the right side. Fifty milliseconds after the offset of the S1 (and thus, $100 \mathrm{~ms}$ after its onset), a target stimulus appeared inside the middle ring on one side. This target stimulus could be either a vertical line $(0.45$ degree long and 0.04 degree wide) or a small ring ( 0.25 degree in diameter and a 0.05-degree wide margin). Both target stimuli were black, with a luminance of less than $0.01 \mathrm{~cd} / \mathrm{m}^{2}$, and lasted $34 \mathrm{~ms}$. Each of them appeared randomly on the same side as the S1 in $25 \%$ of the trials and on the opposite side in $25 \%$ of the trials. The volunteer was instructed to respond as fast as possible to the "vertical line" (the $\mathrm{S} 2+$ ), with the finger on the corresponding side, and not to respond to the "ring" (the S2-). The trial ended with a message lasting $400 \mathrm{~ms}$ at the site of fixation. Reaction time in milliseconds appeared when the volunteer responded between 150 and $600 \mathrm{~ms}$ after the onset of the S2+. The message "CORRECT" appeared when the volunteer did not respond after the S2-. The messages "ANTICIPATED" and "SLOW" were displayed when the volunteer emitted a response before 150 ms after the onset of the S2+/S2- or did not respond until $600 \mathrm{~ms}$ after the onset of the $\mathrm{S} 2+$, respectively. The message "INCORRECT" was displayed when the volunteer responded between 150 and $600 \mathrm{~ms}$ after the onset of the S2- or responded with the wrong index finger. Trials in which the volunteer erred were discarded and repeated later.

The second testing session was similar to the first, with four blocks of 72 trials separated by a resting interval controlled by the volunteer. The messages at the end of the trials were replaced by a green asterisk for correct trials, and a red asterisk for incorrect trials.

Eye movement was not controlled, as previous experiments realized by this laboratory had indicated that the volunteers usually maintain fixation during the trials. It should be noted that the 100-ms stimulus onset asynchrony used did not allow enough time to perform a saccade to the primed location so as to facilitate S2 identification.

\section{Data analysis}

For each volunteer, the block median reaction time and then the mean of the medians of the four blocks were calculated for each condition of the second testing session. The total number of anticipated responses, slow responses, commissioned responses (false alarms) and inverted hand responses for each condition of the second testing session were also evaluated.

Reaction time data were analyzed by repeated measures analysis of variance (ANOVA) and the post hoc Newman-Keuls test. Factors in the ANOVA were the type of $\mathrm{S} 1$ (single, triple and double) and the relative side of the $\mathrm{S} 1$ and the S2+ (same and opposite). The level of significance was set at 0.05 .

\section{Results and Discussion}

Three volunteers were excluded due to an excess of errors (more than $10 \%$ of the trials) and were replaced with three others.

A main effect was obtained for the type of $S 1(F(2,22)=$ 7.186, $\mathrm{P}=0.004)$ and the relative side of $\mathrm{S} 1$ and $\mathrm{S} 2+$ $(F(1,11)=32.165, P<0.001)$ factors; an interaction between these factors also appeared $(F(2,22)=14.349, P<$ $0.001)$. Reaction time was not significantly different between the single S1 and the triple S1 $(P=0.487$; see Figure $2)$. Reaction time was longer when the $S 1$ was double than when it was single $(P=0.009)$ or triple $(P=0.005)$.

Reaction time was shorter when the $\mathrm{S} 2+$ appeared on the same side as the $S 1$ than when it appeared on the opposite side to the $S 1$, for the single $S 1(P<0.001)$, the triple S1 $(P<0.001)$ and the double S1 $(P=0.018)$. When the $\mathrm{S} 2+$ appeared on the same side, reaction time was not significantly different between the single and the triple S1 $(P=0.691)$; it was, however, longer for the double S1 than for the single $S 1(P<0.001)$ and the triple $S 1(P<0.001)$. When the $\mathrm{S} 2+$ appeared on the opposite side, reaction time was not significantly different between the single and the triple S1 $(P=0.193)$, nor the single and the double S1 ( $P$ $=0.221)$, nor even the triple and the double $S 1(P=0.711)$.

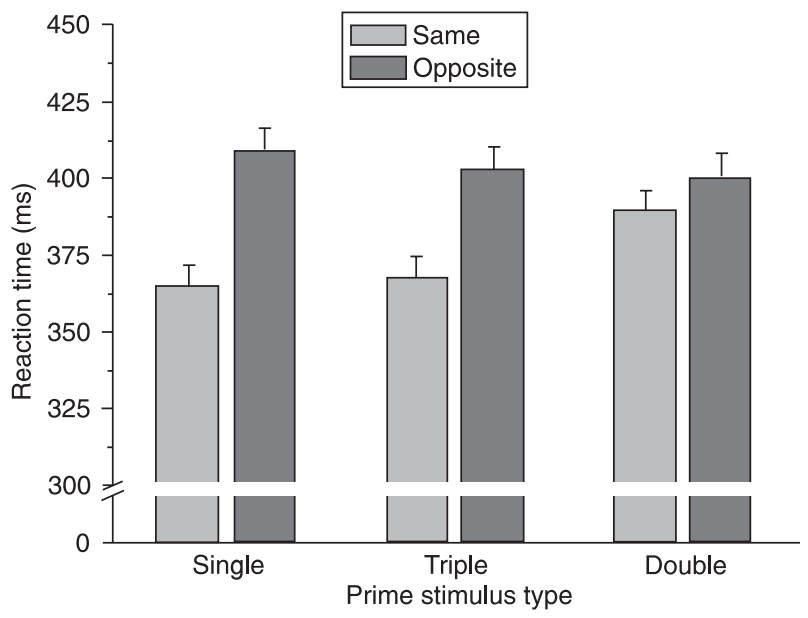

Figure 2. Mean reaction time ( \pm SEM) to the positive target stimulus in the same hemifield and in the opposite hemifield as the prime stimulus in Experiment 1. The prime stimulus could occur in the middle location (single), the superior, middle and inferior locations (triple), or the superior and inferior locations (double). The target stimulus always occurred in the middle location. $\mathrm{N}=12$. 
To alleviate concern that the replacement of three volunteers might have biased the results, extra analyses were performed considering these volunteers. The main results remained unaltered, except for the appearance of a reduction of reaction time to the target stimulus in the middle opposite side location in the double prime stimulus condition, as compared to the single prime stimulus condition.

The percentages of anticipation, omission, commission, and inversion errors were $0.8,4.3,0.9$, and $1.5 \%$, respectively. The relatively small percentages of anticipation, commission and inversion errors do not suggest that the volunteers presented any important tendency to respond to the S1.

As expected, an attentional effect of $44 \mathrm{~ms}$ and $36 \mathrm{~ms}$ occurred in the single $\mathrm{S} 1$ and the triple $\mathrm{S} 1$ conditions, respectively. An attentional effect also occurred in the double $\mathrm{S} 1$ condition but was much smaller than the others (only $11 \mathrm{~ms}$ ). This finding could be taken as evidence that attention extends to the area between two separate primed locations, but is less intense in this area.

It should be noted that the attentional effect reduction in the double $\mathrm{S} 1$ condition was mainly caused by a longer reaction time to the target stimulus in the same side location. This is exactly what would be expected in the case of a partial division of the facilitatory action of attention on stimulus processing.

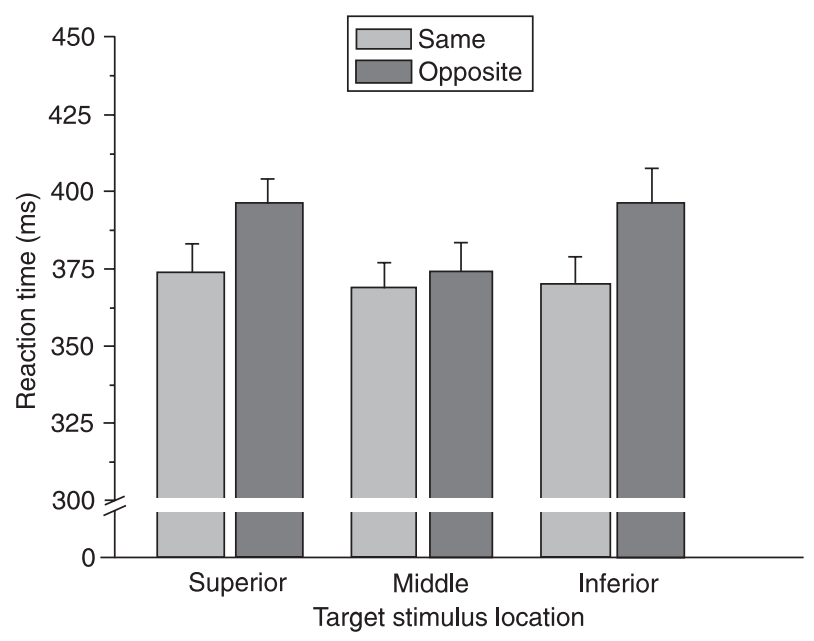

Figure 3. Mean reaction time ( \pm SEM) to the positive target stimulus in the same hemifield and in the opposite hemifield as the prime stimulus in Experiment 2. The prime stimulus always occurred in the superior and inferior locations (double). The target stimulus could occur in the superior, the middle or the inferior location. $\mathrm{N}=12$.

\section{Experiment 2}

Although it is reasonable to assume that automatic attention was focused on the superior and the inferior locations in the double prime stimulus condition (and the triple prime stimulus condition) of the previous experiment, this was not unequivocally demonstrated. This is a critical point for the conclusion that automatic attention was spatially divided in that condition, since the very small effect observed with the double prime stimulus could simply have been due to a weak capture of attention by the superior and inferior components of this stimulus (and those of the triple prime stimulus), possibly caused by the fact that the target stimuli never appeared at their locations. Experiment 2 attempted to confirm that attention was focused on both the superior and inferior locations in a double prime stimulus condition. Only the double prime stimulus was used. The target stimuli could appear in the superior or the inferior location, in addition to the middle location. It was expected that a large attentional effect would appear for the superior and inferior locations of the target stimulus and a small or absent attentional effect would appear for the middle location of the target stimulus.

\section{Methods}

Participants. Twelve different female volunteers with the characteristics described in Experiment 1 were used.

Procedure. The volunteers were tested mostly as described in Experiment 1. The S1 was now of only one type, represented by the darkening of the superior and the inferior rings on one side. The target stimuli appeared equally and randomly in any of the marked locations (see Figure 1).

\section{Data analysis}

The data of the second testing sessions were treated as described in Experiment 1. Factors in the ANOVA were the location of the $\mathrm{S} 2+$ (superior, middle and inferior) and the relative side of the $\mathrm{S} 1$ and the $\mathrm{S} 2+$ (same and opposite).

\section{Results and Discussion}

Five volunteers were excluded due to an excess of errors and were replaced with five others.

A main effect was obtained for the location of S2+ $(F(2,22)=8.920, P=0.001)$ and the relative side of $S 1$ and $\mathrm{S} 2+(F(1,11)=19.571, \mathrm{P}=0.001)$ factors; an interaction between these factors also appeared $(F(2,22)=4.026, P=$ 0.032 ). Reaction time was not significantly different between the superior location and the inferior location $(P=$ 0.713; see Figure 3). Reaction time was shorter when the 
S2+ appeared in the middle location than when it appeared in the superior location ( $P=0.003$ ) or the inferior location $(P=0.002)$.

Reaction time was shorter when the $\mathrm{S} 2+$ appeared on the same side as the $\mathrm{S} 1$ than when it appeared on the opposite side as the $\mathrm{S} 1$, for both the superior location ( $\mathrm{P}=$ $0.003)$ and the inferior location $(P=0.001)$. There was no significant difference between reaction time to the $\mathrm{S} 2+$ on the same side and reaction time to the $\mathrm{S} 2+$ on the opposite side for the middle location $(P=0.887)$. When the S2+ appeared on the same side as the $S 1$, reaction time was not significantly different between the superior and the inferior locations $(P=0.693)$, or the superior and the middle locations $(P=0.842)$, or even the inferior and the middle locations $(P=0.874)$. When the $S 2+$ appeared on the opposite side, reaction time was not significantly different between the superior and the inferior locations ( $P=$ 0.978); it was, however, shorter for the middle location than for the superior location $(P=0.003)$ and the inferior location $(P=0.001)$.

To alleviate concern that the replacement of five volunteers might have biased the results, extra analyses were performed considering these volunteers. The main results remained unaltered.

The percentages of anticipation, omission, commission, and inversion errors were $0.5,5.8,2.6$, and $1.6 \%$, respectively.

An attentional effect occurred for the superior and inferior location conditions, amounting to 23 and $26 \mathrm{~ms}$, respectively. Absolutely no effect occurred for the middle location condition. These results indicate that the two components of the prime stimulus mobilized attention. In addition, they suggest that no attention was mobilized for the middle zone along the horizontal meridian. This reinforces the idea that attention can divide in space.

It must be noted, however, that, in contrast to Experiment 1 , reaction time to the $\mathrm{S} 2+$ in the middle location on the same side as the $\mathrm{S} 1$ was as short as those measured for the $\mathrm{S} 2+$ in the primed locations. On the other hand, reaction time to the $\mathrm{S} 2+$ in the middle location on the opposite side of the S1 was shorter than those measured for the $\mathrm{S} 2+$ in the superior and the inferior locations. These results might be thought to indicate that the facilitatory action of attention was evenly distributed among the three same side locations, but its inhibitory action was restricted to the two locations diametrically opposed to the primed locations. This is quite different from what was considered to have occurred in Experiment 1. In addition, the possibility that the inhibitory action of attention is split does not exactly fit the conventional notion that the entire area surrounding an attended region suffers inhibition (see, for example, Ref. 14).

\section{Experiment 3}

In Experiment 1, a smaller attentional effect was observed in the middle location condition when the S1 was double. The reduction of the attentional effect in this condition was almost exclusively related to an increase in the reaction time in the same side location. In Experiment 2, no attentional effect was found in the middle location condition. The disappearance of the attentional effect was basically due to a decrease in the reaction time in the opposite side location. Thus, although the results of both experiments suggest that attention is faulty in the horizontal zone in between a superior primed location and a corresponding location in the opposite hemifield and an inferior primed location and a corresponding location in the opposite hemifield, they show some important differences.

Experiment 3 aimed at confirming, and eventually extending, the findings of the two previous experiments. The prime stimulus could occur singly, in any of the three locations, or doubly, in the superior and inferior locations, as in Experiment 1. The target stimuli could occur in any of the three locations, as in Experiment 2. Thus, reaction times for the superior, the middle and the inferior locations could be directly compared between the single and the double prime stimulus conditions. It was expected that this might help to explain the above mentioned differences in the results obtained in Experiments 1 and 2 .

\section{Methods}

Participants. Twelve different female volunteers with the characteristics described in Experiment 1 were used.

Procedure. The volunteers were tested mostly as described in Experiment 1. There were three testing sessions; the third session was added to provide the appropriate number of trials per condition. The first session consisted of one block of 72 trials. The second and the third consisted of four blocks of 72 trials. These sessions were performed on different days, with a maximum separating interval of 7 days. The S1 was of four types, represented by the darkening of the superior, middle or inferior ring or both the superior and inferior rings on one side. The target stimuli appeared equally and randomly in any one of the marked locations, with the constraint of always appearing on the same horizontal level as the single S1 (see Figure 1 ). The $S 2+$ occurred in $2 / 3$ of the trials and the $S 2-$ in the remaining $1 / 3$.

Data analysis

Data of the second and third testing sessions were 
grouped and treated as described in Experiment 1. Factors in the ANOVA were the type of $\mathrm{S} 1$ (superior, middle, inferior, and superior-inferior), the location of S2+ (superior, middle, and inferior) and the relative side of $\mathrm{S} 1$ and S2+ (same and opposite).

\section{Results and Discussion}

Five volunteers were excluded due to an excess of errors and were replaced with five others.

A main effect was obtained for the location of $\mathrm{S} 2+$ $(F(2,22)=4.637, P=0.021)$ and the relative side of $\mathrm{S} 1$ and $\mathrm{S} 2+(\mathrm{F}(1,11)=40.838, \mathrm{P}<0.001)$ factors, but not for the type of $S 1(F(1,11)=1.075, P=0.322)$ factor. An interaction occurred between the type of $\mathrm{S} 1$ and the location of the $\mathrm{S} 2+(\mathrm{F}(2,22)=3.718, \mathrm{P}=0.041)$ factors, the type of $\mathrm{S} 1$ and the relative side of $\mathrm{S} 1$ and $\mathrm{S} 2+(\mathrm{F}(1,11)=24.322, \mathrm{P}<$ $0.001)$ factors, and the type of $S 1$, the location of $S 2+$ and the relative side of $S 1$ and $S 2+(F(2,22)=5.260, P=0.014)$ factors. There was no interaction between the location of $\mathrm{S} 2+$ and the relative side of $\mathrm{S} 1$ and $\mathrm{S} 2+(\mathrm{F}(2,22)=1.969$, $P=0.164)$ factors.

Reaction time was not significantly different between the superior location and the inferior location $(P=0.614)$ (see Figure 4). Reaction time was shorter when the S2+ appeared in the middle location than when it appeared in the superior location $(P=0.024)$ or the inferior location ( $P$ $=0.029$ ).

Reaction time to the $\mathrm{S} 2+$ in either the superior or the inferior location did not differ significantly between the

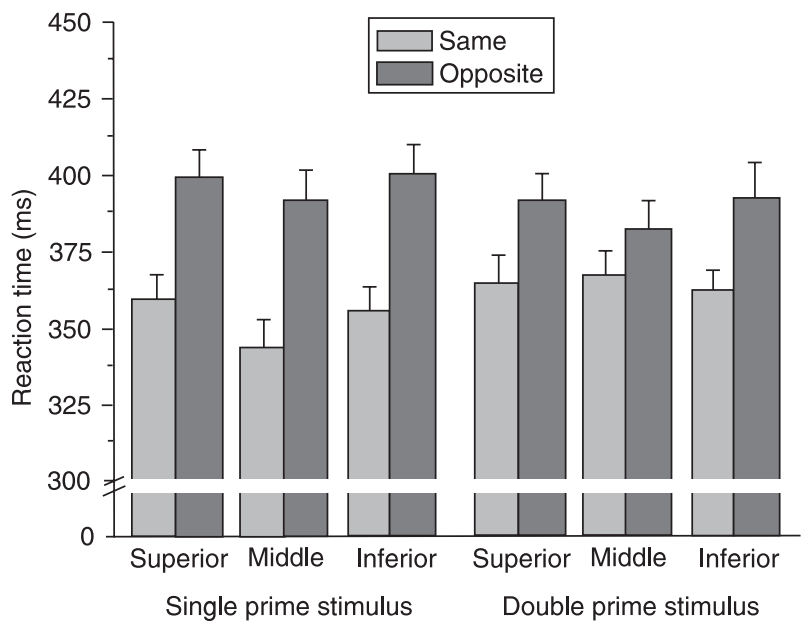

Figure 4. Mean reaction time ( \pm SEM) to the positive target stimulus in the same hemifield and in the opposite hemifield as the prime stimulus in Experiment 3. The prime stimulus could occur in the superior (single), middle (single), inferior (single), or superior and inferior (double) locations. The target stimulus could occur in the superior, the middle or the inferior location. $N=12$. single and the double $\mathrm{S} 1$ (respectively, $\mathrm{P}=0.762$ and $\mathrm{P}=$ $0.697)$. Reaction time to the $S 2+$ in the middle location was longer for the double $S 1$ than for the single $S 1(P=0.002)$.

Reaction time for the same side was longer for the double S1 than for the single S1 $(P=0.002)$. Reaction time for the opposite side was shorter for the double S1 than for the single S1 $(P=0.015)$.

Reaction time to the $\mathrm{S} 2+$ in all three locations was shorter for the same side than for the opposite side, for both the single and double S1. For the same side, reaction time to the $\mathbf{S 2 +}$ in the superior location and the inferior location did not differ significantly between the single S1 and the double $S 1(P=0.457$ and $P=0.402$, respectively); reaction time to the $\mathrm{S} 2+$ in the middle location was longer for the double $S 1$ than that for the single S1 $(P<0.001)$. For the opposite side, reaction time to the $\mathrm{S} 2+$ in the superior location and the inferior location also did not differ significantly between the single S1 and the double S1 $(P=0.422$ and $P=0.208$, respectively); reaction time to the $S 2+$ in the middle location was shorter for the double S1 than for the single S1 $(P=0.042)$.

To alleviate concern that the replacement of five volunteers might have biased the results, extra analyses were performed considering these volunteers. The main results remained unaltered.

The percentages of anticipation, omission, commission, and inversion errors were $0.5,3.9,1.2$, and $0.7 \%$, respectively.

There was an attentional effect for the superior, middle and inferior location of the S2+ of 39,48 , and $43 \mathrm{~ms}$, respectively, when the $\mathrm{S} 1$ was single. There was also an attentional effect for the superior, middle and inferior location of the $S 2+$ of 28,13 , and $31 \mathrm{~ms}$, respectively, when the $\mathrm{S} 1$ was double. The 13-ms attentional effect obtained for the middle location of the S2+ when the S1 was double was smaller than all the other attentional effects, which did not differ significantly between themselves.

The decrease in the attentional effect for the critical condition in the current experiment was produced by both a marked reaction time increase in the middle same side location and a small reaction time reduction in the middle opposite side location, when comparing the double S1 condition with the single $\mathrm{S} 1$ condition. Considering only the double S1 conditions, the decrease in the attentional effect was associated with a reaction time reduction in the middle opposite side location, as compared to the superior and inferior opposite side locations; no reaction time increase in the middle same side location compared to the superior or inferior same side location was apparent.

In general, these results confirm those of Experiments 1 and 2. They provide strong support for the idea that both 
the facilitatory and the diametrically opposed inhibitory action of attention on sensory processing are reduced for a horizontal zone in between a superior primed location and the corresponding location on the opposite hemifield and an inferior primed location and the corresponding location on the opposite hemifield.

The absence of any difference between reaction time in the middle location and reaction time in the superior or the inferior location for the same side double $\mathbf{S 1}$ condition, seen here and also in Experiment 2, might be seen as disturbing for our conclusion of a division of the facilitatory action of attention. Concerns raised by these results are alleviated by taking into account that reaction times in the superior and the inferior locations tend to be higher than reaction time in the middle location. As seen, for the single S1 same side condition of the current experiment, reaction times were longer in the superior and the inferior locations than in the middle location. This might be due to spatial nonuniformities in the visual system (see, for example, Ref. 15). Thus, the simple fact that reaction time in the middle location was similar to those in the superior and inferior locations for the same side condition, here and in Experiment 2, indicates that reaction time increased, presumably as a consequence of partial attention splitting.

\section{General Discussion}

In all three experiments of this study, evidence was obtained that automatic attention, like voluntary attention, may divide in space. Thus, in Experiment 2, no attentional effect occurred for the middle location when a double prime stimulus was presented. In Experiments 1 and 3, the attentional effect produced by this stimulus for the middle location was smaller than that produced by the single or triple prime stimulus for the same location. It is possible that if the distance from the superior and the inferior locations to the middle location had been greater than that used, no attentional effect would have appeared for the middle location also in these two experiments.

The hypothesis of a division of automatic attention in space is reinforced by the consistent increase in reaction time in the same side middle location for the double prime stimulus condition compared to the single prime stimulus condition, in Experiments 1 and 3.

The three locations on each side in all experiments of this study were equidistant from the fixation point. This means that the middle location was somewhat displaced with respect to the axis formed by the superior and the inferior locations. It could be argued that the small or absent effects that were observed for that location in the double priming condition might have been due to the fact that the location was outside a single focus of attention generated by the two superior and inferior components of the prime stimulus. It should be noted, however, that the middle location misalignment was only $0.6^{\circ}$, presumably too small to place it outside this single attentional focus (the target stimuli appeared exactly on a virtual line joining the most peripheral point of the superior ring marker to that of the inferior ring marker).

The possibility that the smaller attentional effect observed for the middle location in the double prime stimulus condition was not associated with a true division of attention, but with the allocation of attention to one of the primed locations in part of the trials and to the other in the other part of the trials should also be considered. The similar reaction times to the target stimulus in the superior or inferior location after presentation of a single prime stimulus and a double prime stimulus in Experiment 3 provide an argument against this alternative. To test the hypothesis in a more specific way, reaction time variance for each of these locations was calculated and compared between the single and the double prime stimulus conditions. No difference was found. A variable allocation of attention to each of the primed locations in the double prime stimulus condition would have necessarily increased reaction time variance, since in the trials in which the location would have been attended, reaction time would have been relatively short, and in the trials in which the location would have been unattended, reaction time would have been relatively long.

Another possible way to explain the smaller attentional effect observed for the middle location in the double prime stimulus condition without assuming any spatial division of attention is to suppose that attention oscillates between the two primed locations during the period of target stimulus presentation. The 34-ms duration of the target stimulus, however, is too short to allow the successful use of this attentional strategy. Castro-Barros et al. (12) demonstrated that it takes between 34 and 50 ms for automatic attention mobilization.

Current accepted knowledge regarding the action of attention on visual processing is that it consists of the facilitation of the attended location and the general inhibition of the remaining locations, even those far away (see, for example, Ref. 14). Based on these assumptions, it could be predicted that attention division in space would be expressed by a reduction or the elimination of its facilitatory effect at a location in between two separated primed locations, accompanied by hardly any change in its inhibitory effect at relatively far locations. The longer reaction time to the target stimulus in a location between the two primed locations, as compared to reaction time to the 
target stimulus in the same location when this was individually primed, observed in Experiments 1 and 3 , is clearly in accordance with this prediction. The shorter reaction time to the target stimulus in the middle location opposed to the double prime stimulus, as compared to the reaction time to the target stimulus in the middle location opposed to the single prime stimulus in Experiment 3, on the other hand, does not conform to this prediction. This unexpected result might be related to the existence of some inverse relationship between the attentional influences (i.e., facilitation and inhibition) in diametrically opposite locations in the visual field or to the existence of a diametrically opposed inhibitory sensory influence, in addition to the inhibitory attentional influence. The well-known inhibitory interactions of the two superior colliculi (16) and of homologous secondary sensory areas in the two cerebral hemispheres (see Ref. 17) could be the basis of this putative sensory influence. The result might also be related to the somewhat larger distance between the primed superior and inferior locations and the opposite middle location than between the primed middle location and the opposite middle location. According to Steinman et al. (14), the inhibitory effect of attention first increases and then decreases as distance from the attended location increases. In counter-argument to these possibilities is the fact that reaction time to the target stimulus in the opposite location in the double stimulus condition did not differ from that in the single stimulus condition in Experiment 1. A critical difference between Experiment 3 and Experiment 1 is the occurrence of the target stimulus in the superior, middle and inferior locations in the former and its occurrence only in the middle location in the latter. Perhaps the contralateral middle location inhibition by a double prime stimulus is adjusted according to the probability of occurrence of the target stimulus in the three opposite side locations. When the target stimulus occurred only in the middle location (Experiment 1) this inhibition would be focused and thus stronger. When the target stimulus could occur in the superior, middle and inferior locations (Experiment 3 ) it would be more diffuse and thus weaker. This is not the first time that a variation in the inhibitory attentional strategy used by the observer has been suggested (e.g., Ref. 9).

Awh and Pashler (9) demonstrated a division of voluntary attention in the presence of distractor stimuli. When these stimuli were not presented, the location intermediary between two relevant locations was fully attended. The authors suggested that the division of the attentional focus probably resulted from the inhibition of non-relevant regions so as to suppress nearby noise (they allowed, however, for an alternative explanation, namely, that the strategy of selectively facilitating the relevant regions could have been adopted so as to increase the signal to noise ratio). In the present series of experiments, there were no distractor stimuli between the superior and the inferior locations, and yet there was an important reduction in the effect produced by the double prime stimulus for the middle location on the same side. This suggests that the influence of automatic attention is represented in an important way by a selective facilitation of the primed locations.

To explain the division of attention observed in their experiments and the absence of any division of attention in the experiments of McCormick and Klein (3), Awh and Pashler (9) considered the cue validity of $80 \%$ that they employed compared to that of $35 \%$ employed by those investigators. They suggested that maintaining more than one attentional focus would be difficult and, because of that, this strategy would be adopted only when it provides a clear advantage to the observer. As the probability of the target stimulus appearing in the primed region in the experiments of the present study was never more than $50 \%$ (in Experiment 1 it was $50 \%$ and in Experiments 2 and 3 it was 33 and $42 \%$, respectively), it would appear that this factor has no importance in the case of automatic attention. It is more likely that the formation of independent foci is the default strategy, as far as this kind of attention is concerned. An interesting question is whether more than two independent foci can be formed. It is possible that when there are more than a certain number of regions to be attended, attention spreads to the whole area instead of forming independent foci.

It is interesting to note that the putative division of automatic attention in the current study occurred in one hemifield. A similar result was described by Awh and Pashler (9) for voluntary attention. These findings might be interpreted as indicating that attentional mechanisms in each hemisphere can control at least two independent foci of facilitation in the contralateral visual hemifield.

Awh and Pashler (9) observed a smaller attentional effect for the inferior than for the superior component of a double vertically oriented cue. They offered no explanation for this difference. In the present study, the attentional effects of the superior and inferior components of the double cue were very similar. If the results of Awh and Pashler (9) are confirmed, this would represent an important distinction between division of voluntary attention and division of automatic attention.

In conclusion, the three experiments of the current study provided evidence that automatic attention, like voluntary attention, may divide in space. Further experiments are required to confirm this possibility and investigate other conditions in which a division of automatic attention might occur. It would also be interesting to determine how many independent attentional foci could be formed. 


\section{References}

1. Desimone R, Duncan J. Neural mechanisms of selective visual attention. Annu Rev Neurosci 1995; 18: 193-222.

2. Posner MI, Snyder CR, Davidson BJ. Attention and the detection of signals. J Exp Psychol 1980; 109: 160-174.

3. McCormick PA, Klein R. The spatial distribution of attention during covert visual orienting. Acta Psychol 1990; 75: 225242.

4. McCormick PA, Klein RM, Johnston S. Splitting versus sharing focal attention: comment on Castiello and Umilta (1992). J Exp Psychol Hum Percept Perform 1998; 24 : 350-357.

5. Heinze HJ, Luck SJ, Munte TF, Gos A, Mangun GR, Hillyard SA. Attention to adjacent and separate positions in space: an electrophysiological analysis. Percept Psychophys 1994; 56: 42-52.

6. Müller HJ, Findlay JM. Sensitivity and criterion effects in the spatial cuing of visual attention. Percept Psychophys 1987; 42: 383-399.

7. Castiello U, Umiltà C. Splitting focal attention. J Exp Psychol Hum Percept Perform 1992; 18: 837-848.

8. Egly R, Homa D. Sensitization of the visual field. $J$ Exp Psychol Hum Percept Perform 1984; 10: 778-793.

9. Awh E, Pashler H. Evidence for split attentional foci. J Exp Psychol Hum Percept Perform 2000; 26: 834-846.
10. Müller MM, Malinowski P, Gruber T, Hillyard SA. Sustained division of the attentional spotlight. Nature 2003; 424: 309312.

11. McMains SA, Somers DC. Processing efficiency of divided spatial attention mechanisms in human visual cortex. $J$ Neurosci 2005; 25: 9444-9448.

12. Castro-Barros BA, Righi LL, Grechi G, Ribeiro-do-Valle LE. Interlateral asymmetry in the time course of the effect of a peripheral prime stimulus. Brain Cogn 2007 (in press).

13. Oldfield RC. The assessment and analysis of handedness: the Edinburgh Inventory. Neuropsychologia 1971; 9: 97113.

14. Steinman BA, Steinman SB, Lehmkuhle S. Visual attention mechanisms show a center-surround organization. Vision Res 1995; 35: 1859-1869.

15. Efron R, Yund EW. Spatial nonuniformities in visual search. Brain Cogn 1996; 31: 331-368.

16. Sprague JM. Interaction of cortex and superior colliculus in mediation of visually guided behavior in the cat. Science 1966; 153: 1544-1547.

17. Fink GR, Driver J, Rorden C, Baldeweg T, Dolan RJ. Neural consequences of competing stimuli in both visual hemifields: a physiological basis for visual extinction. Ann Neurol 2000; 47: 440-446. 